\title{
Spirometry Nose Clip in the Primary Care Setting
}

Daniel Jun Yi Wong ${ }^{1 *}$, Bing Mei Teh ${ }^{2,3}$ and Peter Friedland ${ }^{2,4}$

${ }^{1}$ Monash Health, Moorabbin Hospital, Department of ENT, Monash Health, Bentleigh East, Victoria 3165, Australia

${ }^{2}$ MBBS PhD, Ear Sciences Centre, School of Surgery, The University of Western Australia, Perth, Western Australia, Australia

${ }^{3}$ Department of ENT, Head \& Neck Surgery, Faculty of Medicine, Surgery \& Health Sciences, Monash Health \& Monash University, Victoria, Australia ${ }^{4}$ Sir Charles Gairdner Hospital, Perth, Western Australia, Australia

"Corresponding author: Daniel Jun Yi Wong, ENT, Monash Health, Moorabbin Hospital, Department of ENT, Monash Health, Bentleigh East, Victoria 3165, Australia Rec date: Feb 02, 2016, Acc date: Feb 08, 2016, Pub date: Feb 16, 2016

Copyright: (C) 2016 Wong DJY, et al. This is an open-access article distributed under the terms of the Creative Commons Attribution License, which permits unrestricted use, distribution, and reproduction in any medium, provided the original author and source are credited.

\begin{abstract}
Background: Epistaxis is common clinical presentation to the emergency department. Initial management in mild anterior epistaxis involves manual compression of the anterior aspect of the nose. However, this step is often not done appropriately.

Objective: The purpose of this article is to discuss the benefits of applying nose clips in the management of epistaxis. It can be a simple alternative to providing adequate digital compression in initial management of epistaxis.

Discussion: Swimmer's clips have been previously described in the literature for the management of mild epistaxis. The nose clip described in this article is initially designed for spirometry. This clip is user-friendly, affordable and can be easily applied to provide temporary tamponade.
\end{abstract}

\section{Introduction}

Epistaxis is the one of the commonest presentations to general practice and the emergency department. It has been estimated that up to $60 \%$ of the general population will experience one episode of epistaxis in their lifetime with $6 \%$ seeking medical attention [1]. 90-95\% of epistaxis occurs along the anterior nasal septum at a site known as Little's area where the confluence of vessels (Keisselbach's plexus) lies [2].

Most anterior bleeding is minor and can be managed conservatively. In fact, pinching the anterior aspect of the nose for 15 minutes can provide tamponade to the anterior septal vessels [3] and may alleviate more invasive interventions. However, this important step is often ignored, forgotten or overlooked. Moreover, when digital compression is applied, it is often not applied sufficiently long enough or continuously, as patients often release compression to check for bleeding.

\section{Technique}

Initially meant for nasal occlusion during spirometry testing, this nose clip is made of plastic (Figure 1).

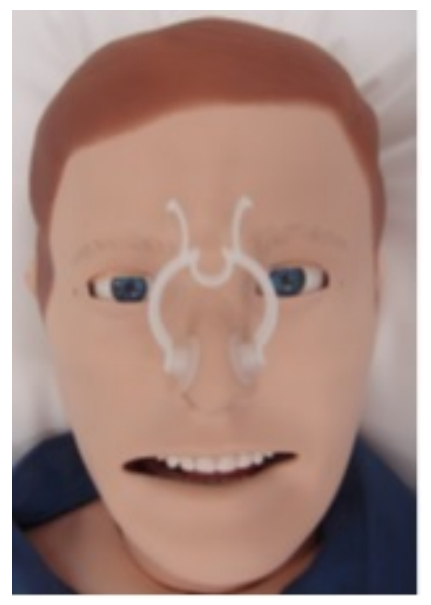

Figure 1: The nose clip is applied to the anterior part of the nose in the event of anterior epistaxis as illustrated here.

Its main body is round with two tips anteriorly which are applied to the nasal alar (Figure 2) whilst the two posterior arms are used to control the opening of the jaws. 
Citation: Wong DJY, Teh BM and Friedland P (2016) Spirometry Nose Clip in the Primary Care Setting. J Gen Practice 4: 230. doi: 10.4172/2329-9126.1000230

Page 2 of 2

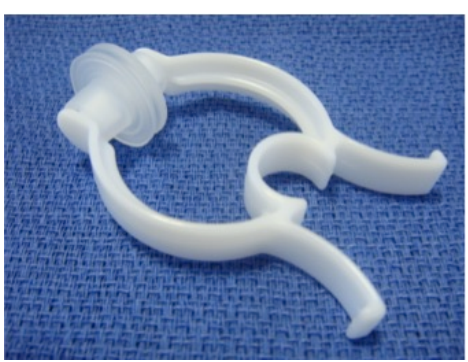

Figure 2: The nose clip consists of a main round body with a soft padding at the end the anterior tips to be applied to the nasal alar. The posterior arms are used to control the opening of the jaws.

Once anterior epistaxis is confirmed by visualisation, this nose clip is applied gently. Pressure should be applied more distally by compressing the nasal alar against the septum instead of along the nasal bones and left continuously for 15-20 minutes. The use of this clip spares nursing staff, the patient and/or the carer from manually compressing the nose, thus allowing the patient to be managed more efficiently. Moreover, the patients' arms are freed, allowing easier access for venipuncture, blood pressure and pulse oximetry monitoring, as well as insertion of intravenous cannulae for fluid resuscitation if required. Instead of compressing the patient's nose for 15 to 20 minutes, medical or nursing staff can now attend to other more important steps in patient care.

These clips are similar to previously published swimmer's clips, which have been used in epistaxis [4,5]. However, unlike the swimmer's clips, these clips are more patient-friendly in terms of design. As they are made of plastic, the pressure exerted is not as forceful, hence providing more comfort for the patients. Moreover, the presence of soft padding at the end of the two arms provides extra comfort to the nose. These lightweight nose clips are also inexpensive (AUD \$0.78 for one), disposable, and portable.
Although they are not designed specifically for epistaxis management, they should be considered in all non-life threatening anterior epistaxis in the immediate setting. Bleeding that ceases with conservative measurers may potentially avoid nasal packing or cauterisation. In addition, patients with known underlying disorders such as hereditary telengiectasia with recurrent epistaxis could apply these nose clips at home and hence avoid frequent visits to the emergency department once properly assessed.

These clips are available in one size only, and can usually fit all patients. They should serve as an adjunct to treatment and other important measures such as appropriate examination, nursing headup, and adequate management of blood pressure. Patients with uninvestigated recurrent epistaxis should also be appropriately worked up.

\section{Conclusion}

In conclusion, the use of nose clips is a simple alternative to digital compression in the initial management of anterior epistaxis. Pilot studies are underway to test the effectiveness of these clips.

\section{References}

1. Marx JA, Hochberger RS (2002) Rosen's Emergency Medicine: Concepts and Clinical Practice.St. Louis, MO: Mosby Inc.

2. Chiu T, Dunn JS (2006) An anatomical study of the arteries of the anterior nasal septum. Otolaryngol Head Neck Surg 134: 33-36.

3. Schlosser RJ (2009) Clinical practice. Epistaxis. N Engl J Med 360: 784-789.

4. Turner P (1996) The swimmer's nose clip in epistaxis. J Accid Emerg Med 13: 134.

5. Vaghela HM (2005) Using a swimmer's nose clip in the treatment of epistaxis in the A\&E department. Accid Emerg Nurs 13: 261-263. 